\title{
Calcium Mandelate
}

National Cancer Institute

\section{Source}

National Cancer Institute. Calcium Mandelate. NCI Thesaurus. Code C77069.

A calcium salt form of mandelic acid, an aromatic alpha hydroxy acid used in the treatment of urinary tract infections and as an oral antibiotic. 